\title{
On the Computation of Euler's Constant
}

\author{
By Dura W. Sweeney
}

1. Introduction. The computation of Euler's constant, $\gamma$, to 3566 decimal places by a procedure not previously used is described. As a part of this computation, the natural logarithm of 2 has been evaluated to 3683 decimal places. A different procedure was used in computations of $\gamma$ performed by J. C. Adams in 1878 [1] and J. W. Wrench, Jr. in 1952 [2], and recently by D. E. Knuth [3]. This latter procedure is critically compared with that used in the present calculation. The new approximations to $\gamma$ and $\ln 2$ are reproduced in extenso at the end of this paper.

2. Evaluation of $\gamma$. A new procedure based upon the expansion of the exponential integral, $-E_{i}(-x)$, was used to evaluate $\gamma$ rather than the classical approach used by Adams, Wrench, and Knuth. This new procedure was chosen so as to avoid the more complex programming required in the computation of high orders of Bernoulli numbers.

The exponential integral is given as

$$
\begin{aligned}
-E_{i}(-x)=\int_{x}^{\infty} \frac{e^{-t} d t}{t}=-\gamma-\ln x & +x-\frac{x^{2}}{2 \cdot 2 !} \\
& +\frac{x^{3}}{3 \cdot 3 !}-\cdots=-\gamma-\ln x+S(x) .
\end{aligned}
$$

Its asymptotic expansion is

$$
-E_{i}(-x)=\int_{x}^{\infty} \frac{e^{-t} d t}{t} \cong \frac{e^{-x}}{x}\left(1-\frac{1}{x}+\frac{2 !}{x^{2}}-\cdots\right)=R(x) .
$$

Equating these and moving $\gamma$ to the left, we have

$$
\gamma \cong S(x)-\ln x-R(x) \text {. }
$$

Since the asymptotic form behaves as $e^{-x} / x$ for large $x$, the difference between $S(x)$ and $\ln x$ will approximate $\gamma$ to the accuracy of the number of leading zeros in the value of $R(x)$.

$$
\text { For } x=8192, \quad R(x)=0.22190 \cdots 10^{-3561} \text {. }
$$

The value of $x$ was chosen as a power of 2 to simplify the calculation of $\ln x$. Also, since a binary computer was to be used, many of the multiplications in the terms of $S(x)$ could be reduced to shifting operations.

3. Method of Computation. The computation of $\ln 2$ is very rapid and straightforward on a binary computer using one of the forms of the expansion

$$
\ln 2=\frac{1}{2}+\frac{1}{2 \cdot 2^{2}}+\frac{1}{3 \cdot 2^{3}}+\cdots .
$$

Received June 29, 1962. 
The computation of $S(x)$ is also straightforward, but requires substantially more computer time since for $x=8192$ almost 30,000 terms are required for convergence, and up to three times the number of digits in the final answer are required during intermediate computations to avoid truncation errors and to compensate for the loss of significant figures arising from subtractions.

The computer program was written to do the computation two different ways to establish the accuracy of the analysis, the programming, and the system operation. Two different binary-to-decimal conversion routines were also used, one with each of the computations.

The first part of the computer run used the following procedure. The individual terms of the expansion,

$$
13 \ln 2=13\left[\frac{5}{1 \cdot 1 \cdot 2^{3}}+\frac{11}{2 \cdot 3 \cdot 2^{5}}+\frac{17}{3 \cdot 5 \cdot 2^{7}} \cdots\right]
$$

were evaluated and summed to form $\ln 8192$. $S(x)$ was evaluated by summing the odd and even terms of the expansion separately to avoid subtractions, thus:

$$
\begin{aligned}
S(x)=\left(x+\frac{x^{3}}{3 \cdot 3 !}+\right. & \cdots \cdots) \\
& -\left(\frac{x^{2}}{2 \cdot 2 !}+\frac{x^{4}}{4 \cdot 4 !}+\cdots\right)=x+\sum D_{2 n+1}-\sum D_{2 n} .
\end{aligned}
$$

The individual terms of these sums were computed from an intermediate value, $C_{2 n}$, as follows:

$$
\begin{gathered}
C_{2 n}=\frac{x^{2 n+1}}{(2 n) !}=\frac{x^{2}}{2 n(2 n-1)} D_{2 n-2}, \text { where } \\
D_{2 n+1}=\frac{C_{2 n}}{(2 n+1)^{2}} \quad \text { and } D_{2 n}=\frac{C_{2 n}}{2 n x} .
\end{gathered}
$$

The second part of the computer run used the following procedure. Ln 8192 was evaluated from the following recursion starting at $n=12,300$ :

$$
\ln 8192=13 B_{1}, \quad B_{n}=\frac{1}{2}\left(\frac{1}{n}+B_{n+1}\right) .
$$

$S(x)$ was evaluated by the following recursion starting at $n=30,000$ :

$$
S(x)=x A_{1}, \quad A_{n}=1-\frac{n x}{(n+1)^{2}} A_{n+1} .
$$

The complete computation was performed on the engineering model of the IBM 7094 in 58 minutes. The first part of the computer run took approximately 20 minutes. The second part took approximately 35 minutes. The remaining time was required for non-overlapped printing and punching of results. The same computation was performed again on an IBM 7090 in 114 minutes as part of the tests of the speed and compatibility of the two systems.

The computed values of $\ln 2$ agreed to 3683 decimal places, and the tabulation is believed accurate to that number of decimals. The value of $\ln 2$ confirms the value calculated by H. S. Uhler [4] to 330 decimal places. 
The computed values of $\gamma$ agreed to the same number of decimal places as $\ln 2$, but the accuracy is limited by the value of $x$ to 3561 decimal places. The value of $R(8192)$ given in (4) was subtracted to give the additional five decimal places shown in parentheses in the tabulation. This value of $\gamma$ is believed accurate to 3566 decimal places and confirms the value calculated by D. E. Knuth to 1270 decimal places.

4. Comparison of Methods. The operating times reported by Knuth presented an opportunity to compare the two methods to determine which might be more useful in extending the value of $\gamma$ to greater accuracy. An estimate of the time required shows that if the expansion of the exponential integral had been used it would have been substantially faster than the classical method for the evaluation of $\gamma$ to 1271 decimal places on the Burroughs 220 .

$$
\begin{aligned}
\text { For } x & =3000, \quad R(x)<10^{-1300}, \quad \text { and } \\
\ln 3000=\frac{7}{8} \ln 10000+ & \frac{1}{2} \ln \left(1-\frac{1}{10}\right) \\
= & \frac{7}{8} \ln 10000-\frac{1}{2}\left(\frac{21}{1 \cdot 2 \cdot 10^{2}}+\frac{43}{3 \cdot 4 \cdot 10^{4}}+\cdots\right) .
\end{aligned}
$$

Knuth reported a time for the evaluation of $\ln 10000$ of approximately 18 minutes. The additional logarithm would take approximately 4 minutes more.

$S(x)$ would require approximately 10,800 terms for convergence and could probably be most efficiently computed as follows:

$$
S(x)=\left(x+\frac{x^{3}}{3 \cdot 3 !}+\cdots\right)-\left(\frac{x^{2}}{2 \cdot 2 !}+\frac{x^{4}}{4 \cdot 4 !}+\cdots\right)=\sum D_{2 n-1}-\sum D_{2 n}
$$

where

$$
D_{2 n-1}=\frac{(2 n-2) x}{(2 n-1)^{2}} D_{2 n-2} \quad \text { and } \quad D_{2 n}=\frac{(2 n-1) x}{(2 n)^{2}} D_{2 n-1} .
$$

The evaluation of each $D_{n}$ would require a multiplication loop, a division loop, and a summation loop which would be used to evaluate each storage word of accuracy for each of the terms required for the convergence of $S(x)$. These loop operations would require less than 10 milliseconds for each word of storage. Since there are 10,800 terms, all that is required is an estimate of the accuracy or number of storage words for each term.

The upper curve in Figure 1 shows the value of $r=\log _{10}\left(3000^{n} / n \cdot n !\right)$. For $n=3000, r$ reaches its maximum value of almost 1300 . At this value of $n$, the value of $D_{n}$ must be known to 2600 decimal places (260 words of storage). To avoid truncation errors, at least 2600 decimal places must be carried for each $n<3000$. As $n$ becomes larger, the required accuracy decreases, reaching 1300 decimal places at $n \cong 8200$, and going to zero at $n \cong 10,800$. This is shown as the difference at a particular $n$ between the upper and lower curves in Figure 1. If the accuracy is carried to 2600 decimal places throughout, as shown by the area between the two curves plus the area outlined by the dotted line, the computation of $S(x)$ would have taken 7.8 hours, i.e.,

$$
\left(\frac{260 \times 10,800 \times .010}{3600}\right)
$$




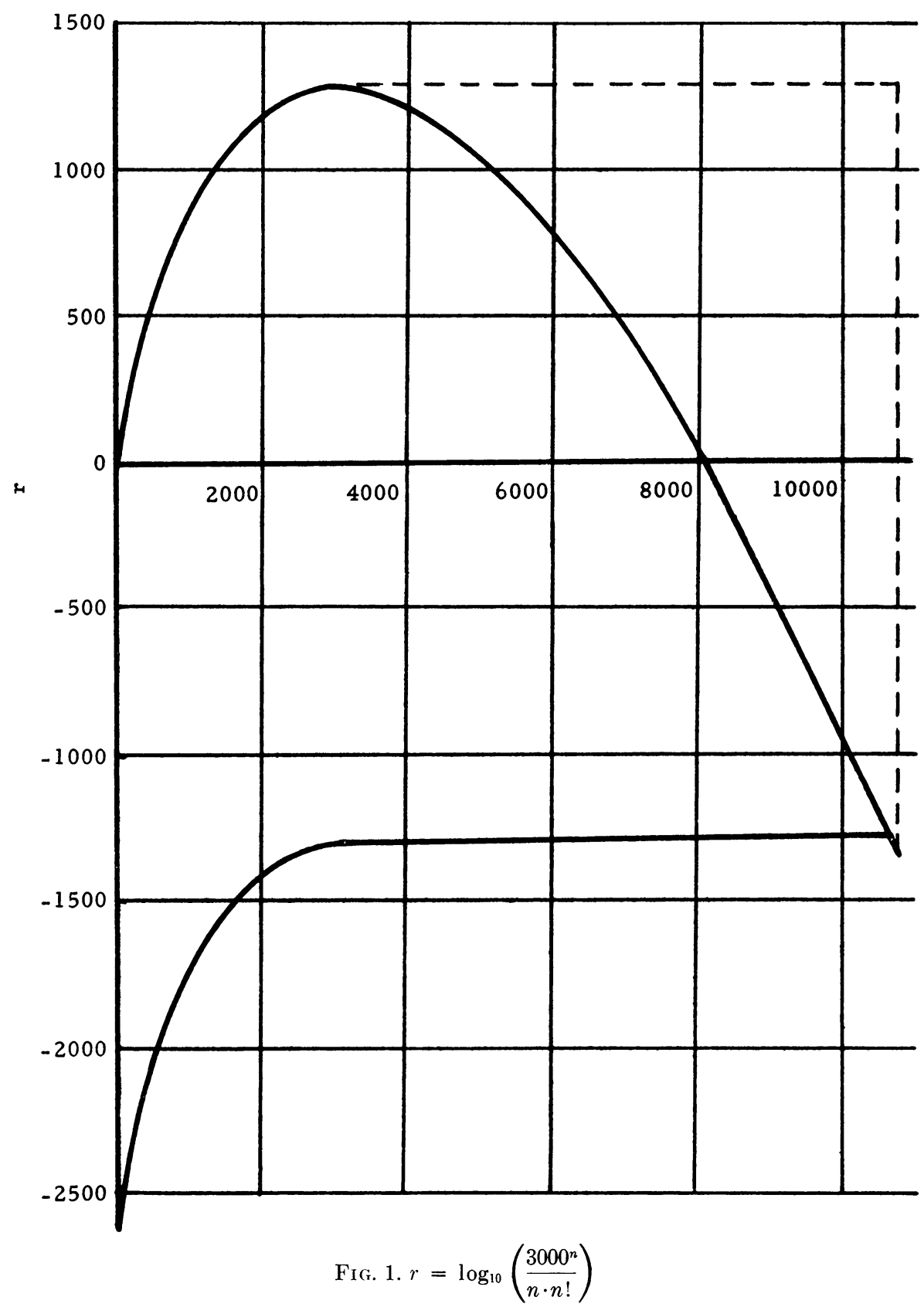

Since the area bounded by the two curves is less than $75 \%$ of the total area considered above, an achievable and still faster time for the evaluation of $S(x)$ would be approximately 5.8 hours. This is to be compared to the approximately 9 hours reported by Knuth for the evaluation of the sum of the first 10,000 reciprocals and the first 250 Bernoulli numbers. When the times for the evaluation of the logarithms are added, the comparison shows that the evaluation of $\gamma$ by this new method would have required about two-thirds of the time reported by Knuth. 
A similar comparison was attempted for the evaluation of $\gamma$ to 3566 decimal places on an IBM 7094 using the classical method. This would have required the evaluation of the sum of the first 65,536 reciprocals and the first 610 Bernoulli numbers. This approach was abandoned since a "good" lower bound of the time required could not be established with reasonable effort because of the complexity in establishing the accuracy (number of words of storage) needed for each of the Bernoulli numbers used in the recursion for evaluating the next higher Bernoulli number. It also appeared that the storage capacity of the system would have been exceeded, requiring additional time and programming complexity. No auxiliary storage is required for the evaluation of $\gamma$ using the expansion of the exponential integral on either computer.

It should be noted that there exists a still faster method which remains to be tried. This method will require additional programming effort, but substantially less computer time will be required. For a given $x$ evaluate $\ln x, S(x)$ and $e^{-x}$ to twice the number of decimal places which would be expected from the value of $R(x)$. Then evaluate the semi-convergent portion of $R(x)$ and multiply by the value of $e^{-x}$. When this value of $R(x)$ is subtracted from $S(x)-\ln x$, the accuracy of $\gamma$ will be extended to that expected from the value of $R(2 x)$. This method will be faster since $S(x)$ will require far fewer terms for convergence to a certain accuracy than $S(2 x)$; e.g., $S(8192)$ will require approximately 36,000 terms for convergence to about 7200 decimal places, while $S(16384)$ will require almost 60,000 terms to achieve the same accuracy.

IBM Data Processing Division

Poughkeepsie, New York

1. J. C. Adams, “On the value of Euler's constant," Proc. Roy. Soc. London, v. 27, 1878, p. $88-94$.

2. J. W. WRENCH, JR., "A new calculation of Euler's constant," MTAC, v. 6, 1952, p. 255.

3. D. E. KNuth, "Euler's constant to 1271 places," Math. Comp., v. 16, 1962, p. 275-281.

4. H. S. UHLER, "Recalculation and extension of the modulus and of the logarithms of 2, 3, 5, 7, and 17," Proc. Nat. Acad. Sci., v. 26, 1940, p. 205-212. 
$\gamma=.57721 \quad 56649015328606065120900824024310421 \quad$ j9335 93992 $\begin{array}{lllllllllll}35988 & 05767 & 23488 & 48677 & 26777 & 66467 & 09369 & 47063 & 29174 & 6749 \tilde{\text { j }}\end{array}$ $\begin{array}{lllllllllll}14631 & 44724 & 98070 & 82480 & 96050 & 40144 & 86542 & 83622 & 41739 & 97644\end{array}$ $\begin{array}{llllllllll}92353 & 62535 & 00333 & 74293 & 73377 & 37673 & 94279 & 25952 & 58247 & 09491\end{array}$

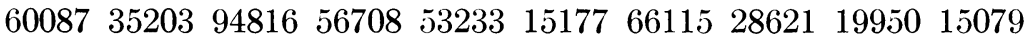

$\begin{array}{llllllllll}84793 & 74508 & 57057 & 40029 & 92135 & 47861 & 46694 & 02960 & 43254 & 21519\end{array}$ $\begin{array}{llllllllllll}05877 & 55352 & 67331 & 39925 & 40129 & 67420 & 51375 & 41395 & 49111 & 68510\end{array}$

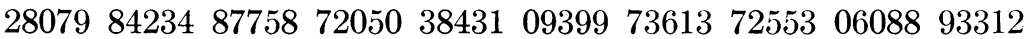
$\begin{array}{llllllllll}67600 & 17247 & 95378 & 36759 & 27135 & 15772 & 26102 & 73492 & 91394 & 07984\end{array}$ $30103417771778088154 \quad 95706 \quad 6107501016 \quad 1916633401 \quad 52278$

$\begin{array}{llllllllll}93586 & 79654 & 97252 & 03621 & 28792 & 26555 & 95366 & 96281 & 76388 & 79272\end{array}$ $\begin{array}{llllllllll}68013 & 24310 & 10476 & 50596 & 37039 & 47394 & 95763 & 89065 & 72967 & 92960\end{array}$ $\begin{array}{llllllllll}10090 & 15125 & 19595 & 09222 & 43501 & 40934 & 98712 & 28247 & 94974 & 71956\end{array}$ $\begin{array}{llllllllll}46976 & 31850 & 66761 & 29063 & 81105 & 18241 & 97444 & 86783 & 63808 & 61749\end{array}$

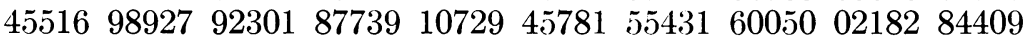
$\begin{array}{lllllllllll}60537 & 72434 & 20328 & 54783 & 67015 & 17739 & 43987 & 00302 & 37033 & 95183\end{array}$ $\begin{array}{llllllllll}28690 & 00155 & 81939 & 88042 & 70741 & 15422 & 27819 & 71652 & 30110 & 73565\end{array}$ $\begin{array}{llllllllll}83396 & 73487 & 17650 & 49194 & 18123 & 00040 & 65469 & 31429 & 99297 & 77956\end{array}$ $\begin{array}{llllllllll}93031 & 00503 & 08630 & 34185 & 69803 & 23108 & 36916 & 40025 & 89297 & 08909\end{array}$

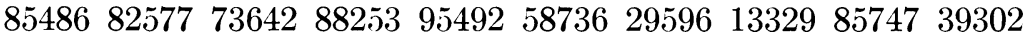

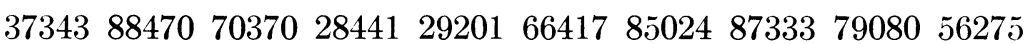
$\begin{array}{llllllllll}49984 & 34590 & 76164 & 31671 & 03146 & 71072 & 23700 & 21810 & 74504 & 44186\end{array}$ $\begin{array}{llllllllll}64759 & 13480 & 36690 & 25532 & 45862 & 54422 & 25345 & 18138 & 79124 & 34573\end{array}$ $\begin{array}{lllllllllll}50136 & 12977 & 82278 & 28814 & 89459 & 09863 & 84600 & 62931 & 69471 & 88714\end{array}$ $9587525492366493520473243641097268276160 \quad 8775950880$

$\begin{array}{llllllllll}95126 & 20840 & 45444 & 77992 & 2991 う & 72482 & 92516 & 25127 & 84276 & 59657\end{array}$ $\begin{array}{llllllllllll}08321 & 46102 & 98214 & 61795 & 19579 & 59095 & 92270 & 42089 & 89627 & 97125\end{array}$ $\begin{array}{lllllllllll}53632 & 17948 & 87376 & 42106 & 60607 & 06598 & 25619 & 90102 & 88075 & 61251\end{array}$ $\begin{array}{llllllllll}99137 & 51167 & 82176 & 43619 & 05705 & 84407 & 83573 & 50158 & 00560 & 77457\end{array}$ $\begin{array}{llllllllll}93421 & 31449 & 88500 & 78641 & 51716 & 15194 & 56570 & 61704 & 32450 & 75008\end{array}$

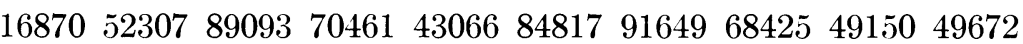
$\begin{array}{lllllllllll}43121 & 83783 & 87535 & 64894 & 95086 & 84541 & 02340 & 60162 & 25085 & 15583\end{array}$ $\begin{array}{lllllllllll}86723 & 49441 & 87880 & 44094 & 07701 & 06883 & 79511 & 13078 & 72023 & 42639\end{array}$ $\begin{array}{lllllllllll}52269 & 20971 & 60885 & 69083 & 82511 & 37871 & 28368 & 20491 & 17892 & 59447\end{array}$

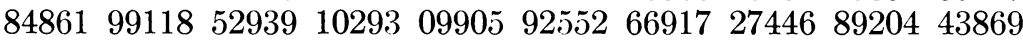

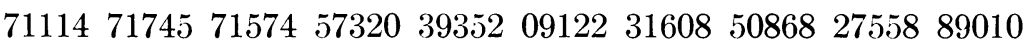
$\begin{array}{llllllllll}94516 & 81181 & 01687 & 49754 & 70969 & 36667 & 12102 & 06304 & 82716 & 58950\end{array}$ $\begin{array}{lllllllllll}49327 & 31486 & 08749 & 40207 & 00674 & 25909 & 18248 & 75962 & 13738 & 42311\end{array}$ $\begin{array}{lllllllllll}44265 & 31350 & 29230 & 31751 & 72257 & 22162 & 83248 & 83811 & 24589 & 57438\end{array}$ $\begin{array}{llllllllll}62398 & 70375 & 76628 & 55130 & 33143 & 92999 & 54018 & 53134 & 14158 & 62127\end{array}$

$\begin{array}{llllllllll}88648 & 07611 & 00301 & 52119 & 65780 & 06811 & 77737 & 63501 & 68183 & 89733\end{array}$ $\begin{array}{lllllllllll}89663 & 98689 & 57932 & 9914 j & 63886 & 44310 & 37060 & 80781 & 74489 & 9579 \tilde{5}\end{array}$ $\begin{array}{llllllllll}83245 & 79418 & 96202 & 60498 & 41043 & 92250 & 78604 & 60362 & 52772 & 60229\end{array}$ $\begin{array}{llllllllllll}19682 & 99586 & 09883 & 39013 & 78717 & 14226 & 91788 & 38195 & 29844 & 56079\end{array}$

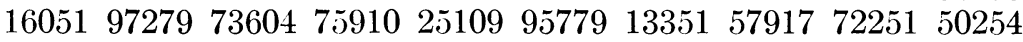

$\begin{array}{llllllllll}92932 & 46325 & 02874 & 76779 & 48421 & 58405 & 07599 & 29040 & 18557 & 64599\end{array}$ $\begin{array}{lllllllllll}01862 & 69267 & 76437 & 26605 & 71176 & 81336 & 55908 & 81554 & 81074 & 70000\end{array}$ $\begin{array}{llllllllll}62336 & 37252 & 88949 & 55463 & 69714 & 33012 & 00791 & 30855 & 52639 & 59549\end{array}$ $\begin{array}{lllllllllll}78230 & 23144 & 03914 & 97404 & 94746 & 82594 & 73208 & 4618 j & 24605 & 87766\end{array}$

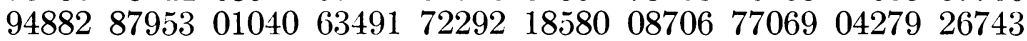




$$
\gamma \text { (continued) }
$$

$\begin{array}{llllllllll}28444 & 69685 & 14971 & 82567 & 80958 & 41654 & 49185 & 14575 & 33196 & 40633\end{array}$ $\begin{array}{lllllllllll}11993 & 73821 & 57345 & 08749 & 88325 & 56088 & 88735 & 28019 & 01915 & 50896\end{array}$ $\begin{array}{lllllllllll}88554 & 68259 & 24544 & 45277 & 28173 & 05730 & 10806 & 06177 & 01136 & 37731\end{array}$ $\begin{array}{lllllllllll}82462 & 92466 & 00812 & 77162 & 10186 & 77446 & 84959 & 51428 & 17901 & 45111\end{array}$ $\begin{array}{llllllllll}94893 & 42288 & 34482 & 53075 & 31187 & 01860 & 97612 & 24623 & 17674 & 9775 \tilde{\jmath}\end{array}$

$\begin{array}{llllllllll}64124 & 61983 & 85640 & 14841 & 23587 & 17724 & 95542 & 24820 & 16151 & 76579\end{array}$ $\begin{array}{lllllllllll}94080 & 62968 & 34242 & 89057 & 25947 & 39269 & 63863 & 38387 & 43805 & 47131\end{array}$ $\begin{array}{llllllllll}96764 & 29268 & 37249 & 07608 & 75073 & 78528 & 37023 & 04686 & 50349 & 05120\end{array}$ $\begin{array}{llllllllll}34227 & 21743 & 66897 & 92848 & 62972 & 90889 & 26789 & 77703 & 26246 & 23912\end{array}$ 26188876530057786274360609444360392809770813383693

$\begin{array}{llllllllll}42355 & 08583 & 94112 & 67092 & 18734 & 41451 & 21878 & 03276 & 15050 & 94780\end{array}$ $\begin{array}{llllllllllll}55466 & 30058 & 68455 & 63152 & 45460 & 53151 & 13252 & 81889 & 10792 & 31491\end{array}$ $\begin{array}{lllllllllll}31103 & 23443 & 02450 & 93345 & 00030 & 76558 & 64874 & 22297 & 17700 & 33178\end{array}$ $\begin{array}{lllllllllll}45391 & 50566 & 94015 & 99884 & 92916 & 09114 & 00294 & 86902 & 08848 & 53816\end{array}$

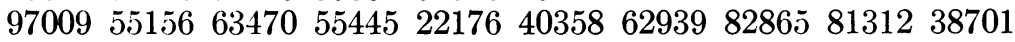

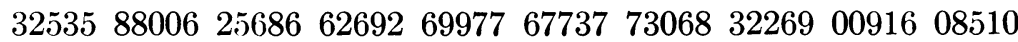
$\begin{array}{lllllllllll}45150 & 02261 & 07180 & 25546 & 59284 & 93894 & 92775 & 95897 & 54076 & 15599\end{array}$ $\begin{array}{lllllllllll}33782 & 64824 & 19795 & 06418 & 68143 & 78817 & 18508 & 85408 & 03679 & 96314\end{array}$ $\begin{array}{llllllllll}23954 & 00919 & 64388 & 75007 & 89000 & 00627 & 99794 & 28098 & 86372 & 99259\end{array}$ 19777650404099220379404276168178371566865306693983

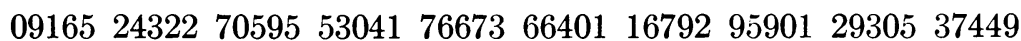
$71830800427(58486)$ 
$\ln 2=.69314 \quad 71805 \quad 59945 \quad 30941 \quad 72321 \quad 21458 \quad 17656 \quad 8075 \check{5} 001343602 \tilde{5}$ $\begin{array}{llllllllll}52541 & 20680 & 00949 & 33936 & 21969 & 69471 & 56058 & 63326 & 99641 & 8687 \tilde{\mathrm{j}}\end{array}$ $\begin{array}{lllllllllll}42001 & 48102 & 05706 & 85733 & 68552 & 02357 & 58130 & 55703 & 26707 & 51635\end{array}$ $\begin{array}{lllllllllll}07596 & 19307 & 27570 & 82837 & 14351 & 90307 & 03862 & 38916 & 73471 & 12335\end{array}$

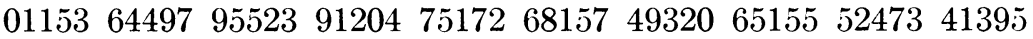

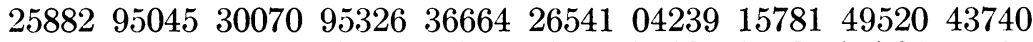
$\begin{array}{llllllllll}43038 & 55008 & 01944 & 17064 & 16715 & 18644 & 71283 & 99681 & 71784 & 54695\end{array}$ $\begin{array}{llllllllll}70262 & 71631 & 06454 & 61502 & 57207 & 40248 & 16377 & 73389 & 63855 & 06952\end{array}$ $\begin{array}{llllllllll}60668 & 34113 & 72738 & 73722 & 92895 & 64935 & 47025 & 76265 & 20988 & 59693\end{array}$ $2019650585547647033067936 \quad 5443254763274495125040606$

$\begin{array}{llllllllll}94381 & 47104 & 68994 & 65062 & 20167 & 72042 & 45245 & 29612 & 68794 & 65461\end{array}$ $\begin{array}{llllllllll}93165 & 17468 & 13926 & 72504 & 10380 & 25462 & 59656 & 86914 & 41928 & 71608\end{array}$ $\begin{array}{lllllllllll}29380 & 31727 & 14367 & 78265 & 48775 & 66485 & 08567 & 40776 & 48451 & 46443\end{array}$ $\begin{array}{llllllllllll}99404 & 61422 & 60319 & 30967 & 35402 & 57444 & 60703 & 08096 & 08504 & 74866\end{array}$ $3852313818167675143866747664789088143714 \quad 1985494231$

$\begin{array}{llllllllll}51997 & 35488 & 03751 & 65861 & 27535 & 29166 & 10007 & 10535 & 58249 & 87941\end{array}$ $\begin{array}{llllllllll}47295 & 09293 & 11389 & 71559 & 98205 & 65439 & 28717 & 00072 & 18085 & 76102\end{array}$ $\begin{array}{llllllllll}52368 & 89213 & 24497 & 13893 & 20378 & 43935 & 30887 & 74825 & 97017 & 15591\end{array}$ $\begin{array}{lllllllllll}07088 & 23683 & 62758 & 98425 & 89185 & 35302 & 43634 & 21436 & 70611 & 89236\end{array}$ $\begin{array}{llllllllll}78919 & 23723 & 14672 & 32172 & 05340 & 16492 & 56872 & 74778 & 23445 & 35347\end{array}$

$\begin{array}{llllllllll}64811 & 49418 & 64238 & 67767 & 74406 & 06956 & 26573 & 79600 & 86707 & 62571\end{array}$ $\begin{array}{llllllllll}99184 & 73402 & 26514 & 62837 & 90488 & 30620 & 33061 & 14463 & 00737 & 19489\end{array}$ $\begin{array}{lllllllllll}00274 & 36439 & 65002 & 58093 & 65194 & 43041 & 19115 & 06080 & 94879 & 30678\end{array}$ $\begin{array}{lllllllllll}65158 & 87090 & 06052 & 03468 & 42973 & 61938 & 41289 & 65255 & 65396 & 86022\end{array}$ $\begin{array}{lllllllllll}19412 & 29242 & 07574 & 32175 & 74890 & 97705 & 75268 & 71158 & 17051 & 13700\end{array}$

$\begin{array}{llllllllll}91589 & 42665 & 47859 & 59648 & 90653 & 05846 & 02586 & 68382 & 94002 & 28330\end{array}$ $\begin{array}{lllllllllll}05382 & 07400 & 56770 & 53046 & 78700 & 18416 & 24044 & 18833 & 23279 & 83863\end{array}$ $\begin{array}{lllllllllll}49001 & 56312 & 18895 & 60650 & 55315 & 12721 & 99398 & 33203 & 07514 & 08426\end{array}$ $\begin{array}{llllllllllll}09147 & 90012 & 65168 & 24344 & 3893 j & 72472 & 78820 & 54862 & 71552 & 74187\end{array}$

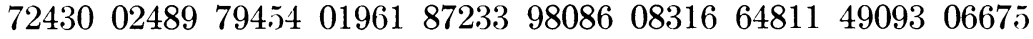

$\begin{array}{llllllllll}19339 & 31289 & 04316 & 41370 & 68139 & 77764 & 98176 & 97486 & 89038 & 87789\end{array}$ $\begin{array}{llllllllll}99129 & 65036 & 19270 & 71088 & 92641 & 05230 & 92478 & 39173 & 73501 & 22984\end{array}$ $\begin{array}{llllllllll}24204 & 99568 & 93599 & 22066 & 02204 & 65494 & 15106 & 13918 & 78857 & 44245\end{array}$ $\begin{array}{lllllllllll}57751 & 02068 & 37030 & 86661 & 94808 & 96412 & 18680 & 77902 & 08181 & 58858\end{array}$ $\begin{array}{llllllllll}00016 & 88115 & 97305 & 61866 & 76199 & 18739 & 52007 & 66719 & 21459 & 22367\end{array}$

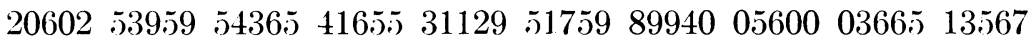
$\begin{array}{lllllllllll}56905 & 12459 & 26825 & 74394 & 64831 & 68332 & 62490 & 18038 & 24240 & 82423\end{array}$ $\begin{array}{llllllllll}14523 & 06140 & 96380 & 57007 & 02551 & 38770 & 26817 & 85163 & 06902 & 55137\end{array}$ $\begin{array}{llllllllll}03234 & 05380 & 21450 & 19015 & 37402 & 95099 & 42262 & 99577 & 96474 & 27138\end{array}$

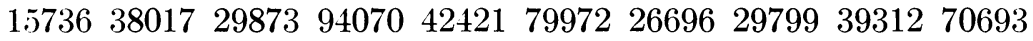

$\begin{array}{llllllllll}5747 & 40493 & 38653 & 08797 & 58721 & 69964 & 51294 & 46491 & 88377 & 11567\end{array}$ $\begin{array}{lllllllllll}01678 & 59880 & 49818 & 38896 & 78413 & 49383 & 14014 & 07316 & 64727 & 65327\end{array}$ $\begin{array}{lllllllllll}63591 & 92335 & 11233 & 38933 & 87095 & 13209 & 05927 & 21854 & 71328 & 97547\end{array}$ $\begin{array}{lllllllllll}07978 & 91384 & 44546 & 66761 & 92702 & 88553 & 34234 & 29899 & 32180 & 37691\end{array}$ 54973 $34026 \quad 75467 \quad 5887323677834291619181043 \quad 01160 \quad 91695$

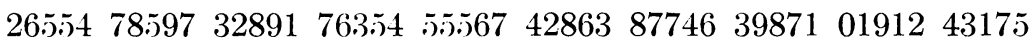
$\begin{array}{llllllllll}425.58 & 88301 & 20677 & 92102 & 80341 & 20687 & 97591 & 43081 & 28330 & 72303\end{array}$ $\begin{array}{lllllllllll}00883 & 49470 & 57924 & 96591 & 00586 & 00123 & 41561 & 75741 & 32724 & 65943\end{array}$ $\begin{array}{lllllllllll}06843 & 54652 & 11135 & 02154 & 43415 & 39955 & 38185 & 65227 & 50221 & 42456\end{array}$ $\begin{array}{llllllllll}64400 & 06276 & 18330 & 32064 & 72725 & 72197 & 51529 & 08278 & 56842 & 13207\end{array}$ 
In 2 (continued)

$\begin{array}{llllllllll}95988 & 63896 & 72771 & 19552 & 21881 & 90466 & 03957 & 00977 & 47065 & 12619\end{array}$

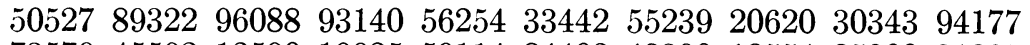
$\begin{array}{llllllllll}73579 & 45592 & 12590 & 19925 & 59114 & 84402 & 42390 & 12554 & 25900 & 31295\end{array}$ $\begin{array}{lllllllllll}37051 & 92206 & 15064 & 34583 & 78787 & 30020 & 35414 & 42178 & 57580 & 13236\end{array}$ $\begin{array}{llllllllll}45166 & 07099 & 14383 & 14500 & 49858 & 96688 & 57722 & 21486 & 52882 & 16941\end{array}$

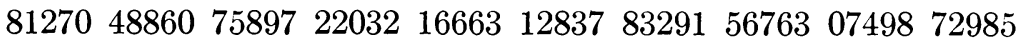
$\begin{array}{lllllllllll}74638 & 92826 & 93735 & 09840 & 77804 & 93950 & 04933 & 99876 & 26475 & 50703\end{array}$ $\begin{array}{llllllllll}16221 & 61390 & 34845 & 29942 & 49172 & 48373 & 40613 & 66226 & 38349 & 36811\end{array}$ $\begin{array}{lllllllllll}16841 & 67056 & 92521 & 47513 & 83930 & 63845 & 53718 & 62687 & 79732 & 88955\end{array}$

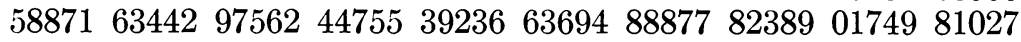

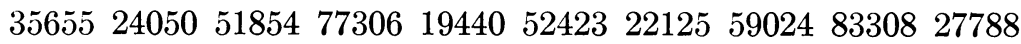
$\begin{array}{lllllllllll}88890 & 59629 & 11972 & 99545 & 74415 & 62451 & 24859 & 26831 & 12607 & 46797\end{array}$ $\begin{array}{llllllllll}28163 & 80902 & 50005 & 65599 & 91461 & 28332 & 54358 & 11140 & 48482 & 06064\end{array}$ $\begin{array}{llllllllllll}08242 & 24792 & 40385 & 57647 & 62350 & 31100 & 32425 & 97091 & 42501 & 11461\end{array}$ 55848306700125831821915347207474111940098355732728

$\begin{array}{llllllllll}26144 & 27382 & 13970 & 70477 & 95625 & 96705 & 79023 & 03384 & 80617 & 13455\end{array}$ $\begin{array}{lllllllllll}55368 & 55375 & 81065 & 74973 & 44479 & 22511 & 19654 & 61618 & 27896 & 01006\end{array}$ $\begin{array}{llllllllll}85129 & 65395 & 47965 & 86637 & 83522 & 47362 & 45460 & 93585 & 03605 & 06784\end{array}$ $\begin{array}{llllllllll}14391 & 14452 & 31457 & 78033 & 59179 & 21127 & 95570 & 50555 & 54514 & 38788\end{array}$ $818815351948593446724642949864 \quad 05062651842447539566$ $\begin{array}{llllllllll}37833 & 73482 & 20753 & 32944 & 81306 & 49336 & 03546 & 10101 & 77464 & 93267\end{array}$ $\begin{array}{lllllllllll}87716 & 71986 & 12073 & 96832 & 01235 & 96077 & 29024 & 68304 & 59403 & 13056\end{array}$ $\begin{array}{lllllllllll}37763 & 13240 & 10804 & 20285 & 43590 & 26945 & 09403 & 07400 & 14933 & 95076\end{array}$ $\begin{array}{llllll}73160 & 28502 & 8697303187 & 18239 & 98433 & 525\end{array}$ 